\title{
Approximate analytic solutions of the modified Kawahara equation with homotopy analysis method
}

\section{Muhammet Kurulay*}

"Correspondence:

mkurulay@yildiz.edu.tr Faculty of Art and Sciences,

Department of Mathematics, Yildiz Technical University, Davutpasa,

İstanbul, 34210, Turkey

\begin{abstract}
In this paper, we applied the homotopy analysis method (HAM) to solve the modified Kawahara equation. Numerical results demonstrate that the methods provide efficient approaches to solving the modified Kawahara equation. It is shown that the method, with the help of symbolic computation, is very effective and powerful for discrete nonlinear evolution equations in mathematical physics.
\end{abstract}

Keywords: the modified Kawahara equation; homotopy analysis method

\section{Introduction}

In the past several decades, the investigation of traveling-wave solutions for nonlinear equations has played an important role in the study of nonlinear physical phenomena. Nonlinear wave phenomena of dispersion, dissipation, diffusion, reaction, and convection are very important in nonlinear wave equations. In recent years, many authors paid attention to study solitonic solutions of nonlinear equations by using a variety of powerful methods such as the variational iteration method (VIM) $[1,2]$ and homotopy perturbation method (HPM) [3, 4]. Exp-function method [5-8], sine-cosine method [9], and homogeneous balance method [10-12] have been proposed for obtaining exact and approximate analytic solutions.

The aim of this paper is to directly apply the optimal HAM $[13,14]$ to reconsider the traveling-wave solution of the Kawahara equation. The method used here contains three convergence-control parameters to accelerate the convergence of homotopy series solution. The optimal convergence-control parameters can be determined by minimizing the averaged residual error. The results obtained in this paper show that the solutions given by the optimal HAM give much better approximations and converge much faster than those given by the usual HAM. The homotopy analysis method (HAM) [15-18] is a general analytic approach to get series solutions of various types of nonlinear equations, including algebraic equations, ordinary differential equations, partial differential equations, differential-integral equations, differential-difference equation, and coupled equations of them.

\section{The homotopy analysis method}

In this paper, we use the homotopy analysis method to solve the problem.

0 2012 Kurulay: licensee Springer. This is an Open Access article distributed under the terms of the Creative Commons Attribution License (http://creativecommons.org/licenses/by/2.0), which permits unrestricted use, distribution, and reproduction in any medium, provided the original work is properly cited. 
This method was proposed by the Chinese mathematician Liao [15]. We apply Liao's basic ideas to the nonlinear partial differential equations. Let us consider the nonlinear partial differential equation

$$
N D(u(x, t))=0 \text {. }
$$

Based on the constructed zero-order deformation equation, we give the following zeroorder deformation equation in the similar way:

$$
(1-q) L\left(U(x, t ; q)-u_{0}(x, t)\right)=q h N D(U(x, t ; q)), \quad q \in[0,1], h \neq 0 .
$$

$L$ is an auxiliary linear integer-order operator and it possesses the property $L(C)=0 . U$ is an unknown function. Expanding $U$ in Taylor series with respect to $q$, one has

$$
U(x, t ; q)=u_{0}(x, t)+\sum_{m=1}^{\infty} u_{m}(x, t) q^{m}
$$

where

$$
u_{m}(x, t)=\left.\frac{\partial^{m} U(x, t ; q)}{\partial q^{m}}\right|_{q=0}
$$

As $h=-1$, Eq. (2.2) becomes

$$
(1-q) L\left(U(x, t ; q)-u_{0}(x, t)\right)+q N D U(x, t ; q)=0, \quad q \in[0,1]
$$

which is used mostly in the homotopy perturbation method (HPM) [19-22]. Thus, HPM is a special case of HAM.

Differentiating the equation $m$ times with respect to the embedding parameter $q$ and then setting $q=0$ and finally dividing them by $m$ !, we have the $m$ th-order deformation equation

$$
L\left[u_{m}(x, t)-\chi_{m} u_{m-1}(x, t)\right]=h R_{m}\left[\vec{u}_{m-1}(x, t)\right]
$$

where

$$
R_{m}\left[\vec{u}_{m-1}(x, t)\right]=\left.\frac{1}{(m-1) !} \frac{\partial^{m-1} N D(U(x, t ; q))}{\partial q^{m-1}}\right|_{q=0}
$$

and

$$
\chi_{m}= \begin{cases}0, & m \leq 1 \\ 1, & m>1\end{cases}
$$

These equations can be easily solved with software such as Maple, Matlab and so on.

The $m$ th-order deformation Eq. (2.6) is linear, and thus can be easily solved, especially by means of a symbolic computation software such as Maple, Matlab and so on. 


\section{Test problem}

We first consider the modified Kawahara equation [23]

$$
u_{t}+u^{2} u_{x}+p u_{x x x}+q u_{x x x x x}=0
$$

where $p, q$ are nonzero real constants. We solve the nonlinear partial differential equation with the HAM method. We consider Eq. (3.1) with initial condition

$$
u(x, 0)=\frac{3 p}{\sqrt{-10 q}} \sec h^{2}(K x)
$$

where $K=\frac{1}{2} \sqrt{\frac{-p}{5 q}}$ is constant. The exact solution is given for modified Kawahara equation by [23]

$$
u(x, t)=\frac{3 p}{\sqrt{-10 q}} \sec h^{2}(K(x-c t))
$$

with $c=\frac{25 q-4 p^{2}}{25 q}$.

Furthermore, Eq. (3.1) suggests defining the nonlinear fractional partial differential operator

$$
\begin{aligned}
N D(u(x, t ; q))= & u_{t}(x, t ; q)+(u(x, t ; q))^{2} u_{x}(x, t ; q) \\
& +p u_{x x x}(x, t ; q)+q u_{x x x x x}(x, t ; q) .
\end{aligned}
$$

Applying the above definition, we construct the zeroth-order deformation equation

$$
(1-q) L\left(u(x, t ; q)-u_{0}(x, t)\right)=h q N D u(x, t ; q) .
$$

For $q=0$ and $q=1$ respectively, we can write

$$
u(x, t ; 0)=u_{0}(x, t)=u(x, 0), \quad v(x, t ; 1)=u(x, t) .
$$

According to Eqs. (2.6)-(2.7), we gain the $m$ th-order deformation equation

$$
L\left(u_{m}(x, t)-x_{m} u_{m-1}(x, t)\right)=h N R\left(\vec{u}_{m-1}(x, t)\right)
$$

where

$$
\begin{aligned}
N R\left(u_{m}(x, t)\right)= & D_{t} u_{m-1}(x, t)+\sum_{i=0}^{j} u_{i} u_{j-i} \sum_{j=0}^{m-1}\left(u_{m-1-j}\right)_{x}(x, t) \\
& +p\left(u_{m-1}\right)_{x x x}(x, t)+q\left(u_{m-1}\right)_{x x x x x}(x, t) .
\end{aligned}
$$

Now, the solution of Eq. (3.7) for $m \geq 1$ becomes

$$
u_{m}(x, t)=\chi_{m} u_{m-1}(x, t)+h L^{-1} N R\left[\vec{u}_{m-1}(x, t)\right] .
$$


Table 1 The numerical results for the approximate solutions obtained by HPM [4] and HAM in comparison with the exact solutions of (3.1)

\begin{tabular}{rllll}
\hline $\boldsymbol{X}$ & Time & HPM & HAM & Exact solution \\
\hline-0.5 & 0.02 & $9.474889415 \mathrm{e}-4$ & $9.474889415 \mathrm{e}-4$ & $9.474889415 \mathrm{e}-4$ \\
-2.5 & 0.04 & $9.483868961 \mathrm{e}-4$ & $9.483868961 \mathrm{e}-4$ & $9.483773375 \mathrm{e}-4$ \\
0.0 & 0.06 & $9.486832980 \mathrm{e}-4$ & $9.486832980 \mathrm{e}-4$ & $9.486831272 \mathrm{e}-4$ \\
2.5 & 0.08 & $9.483868961 \mathrm{e}-4$ & $9.483868961 \mathrm{e}-4$ & $9.484055589 \mathrm{e}-4$ \\
5.0 & 1.0 & $9.474984315 \mathrm{e}-4$ & $9.474984315 \mathrm{e}-4$ & $9.475453144 \mathrm{e}-4$ \\
\hline
\end{tabular}

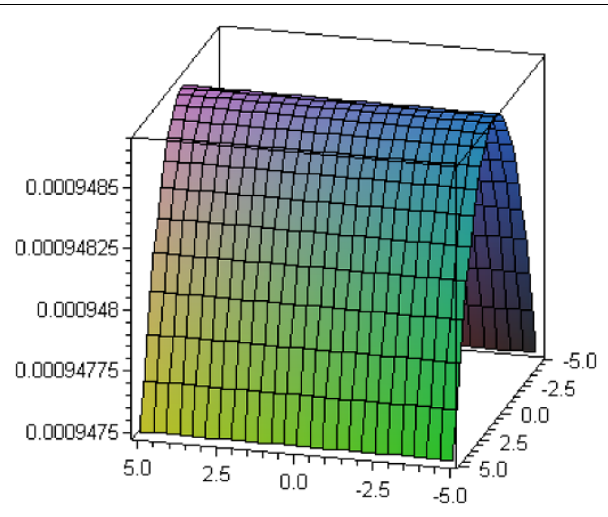

Figure 1 The surface of the exact solutions to Eq. (3.1) obtained in this work.

From Eqs. (3.1), (3.6), and (3.9), for $h=-1$, we now successively get

$$
\begin{aligned}
u(x, t)= & \frac{27}{5} \frac{p^{3} \sec h(K x)^{6} \tanh (k x) k t}{\sqrt{-10 q} q}+p\left(-\frac{24 p \sec h(K x)^{2} \tanh (k x)^{3} k^{3}}{\sqrt{-10 q}}\right. \\
& \left.+\frac{48 p \sec h(K x)^{2} \tanh (k x) k^{3}\left(1-\tanh (k x)^{2}\right)}{\sqrt{-10 q}}\right) t \\
& +\left(\frac{96 p \operatorname{sech}(K x)^{2} \tanh (k x)^{5} k^{5}}{\sqrt{-10 q}}+\frac{1,248 p \sec h(K x)^{2} \tanh (k x)^{3} k^{5}\left(1-\tanh (k x)^{2}\right)}{\sqrt{-10 q}}\right. \\
& \left.-\frac{816 p \sec h(K x)^{2} \tanh (k x) k^{5}\left(1-\tanh (k x)^{2}\right)^{2}}{\sqrt{-10 q}}\right) q t .
\end{aligned}
$$

As shown in Table 1, we note through the results of the preceding table that the solutions we have obtained are very precise and that we have compared our solution (HAM) to HPM and exact solution. HAM is easily more than the other method. It is obvious that two components only were sufficient to determine the exact solution of Eq. (3.1). Figures 1 and 2 show the evolution results. From Figures 1 and 2, it is easy to conclude that the solution continuously depends on the derivative. Where, Figures 1 and 2 are approximation and exact solution respectly. The exact solution of this test problem is as follows [23]:

$$
u(x, t)=\frac{3 p}{\sqrt{-10 q}} \sec h^{2}(K(x-c t)) .
$$

\section{Conclusions}

In this paper, we applied the homotopy analysis method to the Kawahara equation. The homotopy analysis method was successfully used to obtain the exact solutions of Kawa- 


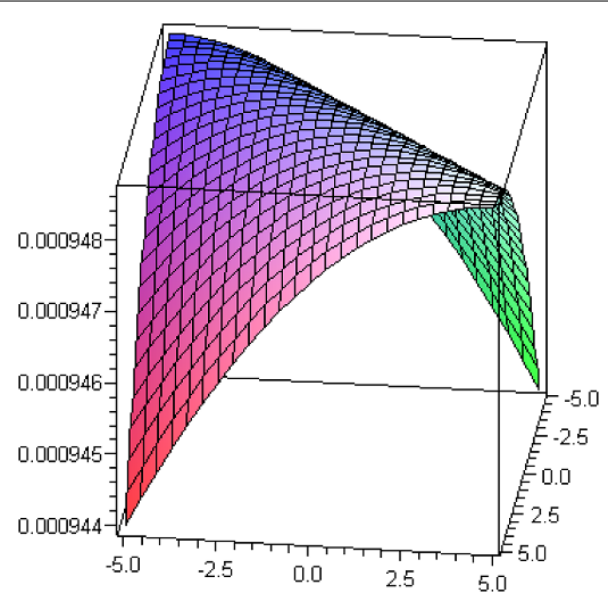

Figure 2 The approximation solutions to Eq. (3.1) obtained in this work.

hara equation. As a result, some new generalized solitary solutions with parameters are obtained. It may be important to explain some physical phenomena by setting the parameters as special values. Finally, the method is straightforward, concise, and is a powerful mathematical method for solving nonlinear problems.

\section{Competing interests}

The author declares that they have no competing interests.

\section{Received: 12 August 2012 Accepted: 28 September 2012 Published: 11 October 2012}

\section{References}

1. He, JH: Variational iteration method for delay differential equations. Commun. Nonlinear Sci. Numer. Simul. 2(4), 235-236 (1997)

2. $\mathrm{He}, \mathrm{JH}$ : Approximate solution of nonlinear differential equations with convolution product nonlinearities. Comput. Methods Appl. Mech. Eng. 167(1-2), 69-73 (1998)

3. He, JH: Homotopy perturbation method: a new nonlinear analytical technique. Appl. Math. Comput. 135, 73-79 (2003)

4. Jin, L: Application of variational iteration method and homotopy perturbation method to the modified Kawahara equation. Math. Comput. Model. 49, 573-578 (2009)

5. He, J-H, Wu, X-H: Exp-function method for nonlinear wave equations. Chaos Solitons Fractals 30, $700-708$ (2006)

6. $\mathrm{He}, \mathrm{JH}, \mathrm{Abdou}, \mathrm{MA}$ : New periodic solutions for nonlinear evolution equations using Exp-function method. Chaos Solitons Fractals 34(5), 1421-1429 (2007)

7. Abbasbandy, S: Homotopy analysis method for the Kawahara equation. Nonlinear Anal., Real World Appl. 11, 307-312 (2010)

8. Yusufoglu, E, Bekir, A: Symbolic computation and new families of exact travelling solutions for the Kawahara and modified Kawahara equations. Comput. Math. Appl. 55, 1113-1121 (2008)

9. Wazwaz, AM: The tanh and the sine-cosine methods for a reliable treatment of the modified equal width equation and its variants. Commun. Nonlinear Sci. Numer. Simul. 11(2), 148-160 (2006)

10. Fan, E, Zhang, H: A note on the homogeneous balance method. Phys. Lett. A 246, 403-406 (1998)

11. Senthilvelan, M: On the extended applications of homogeneous balance method. Appl. Math. Comput. 123, 381-388 (2001)

12. Wang, ML: Exact solutions for a compound KdV-Burgers equation. Phys. Lett. A 213, 279-287 (1996)

13. Dehghan, M, Manafian, J, Saadatmandi, A: Solving nonlinear fractional partial differential equations using the homotopy analysis method. Numer. Methods Partial Differ. Equ. (2009). doi:10.1002/num.20460

14. Assas, LMB: New exact solutions for the Kawahara equation using Exp-function method. J. Comput. Appl. Math. 233, 97-102 (2009)

15. Liao, SJ: The proposed homotopy analysis technique for the solution of nonlinear problems. PhD thesis, Shanghai Jiao Tong University, Shanghai, China (1992)

16. Liao, SJ: An approximate solution technique not depending on small parameters: a special example. Int. J. Non-Linear Mech. 30(3), 371-380 (1995)

17. Liao, SJ: A kind of approximate solution technique which does not depend upon small parameters - II. An application in fluid mechanics. Int. J. Non-Linear Mech. 32(5), 815-822 (1997)

18. Liao, SJ: Beyond Perturbation: Introduction to the Homotopy Analysis Method. CRC Series: Modern Mechanics and Mathematics, vol. 2. Chapman \& Hall/CRC, Boca Raton (2004) 
19. Dehghan, M, Shakeri, F: Use of He's homotopy perturbation method for solving a partial differential equation arising in modeling of flow in porous media. J. Porous Media 11, 765-778 (2008)

20. Shakeri, F, Dehghan, M: Solution of the delay differential equations via homotopy perturbation method. Math. Comput. Model. 48, 486-498 (2008)

21. Dehghan, M, Shakeri, F: Solution of an integro-differential equation arising in oscillating magnetic fields using He's homotopy perturbation method. Prog. Electromagn. Res. 78, 361-376 (2008)

22. Saadatmandi, A, Dehghan, M, Eftekhari, A: Application of He's homotopy perturbation method for non-linear system of second-order boundary value problems. Nonlinear Anal., Real World Appl. 10, 1912-1922 (2009)

23. Daoreji, S: New exact travelling wave solutions for the Kawahara and modified Kawahara equations. Chaos Solitons Fractals 19, 147-150 (2004)

doi:10.1186/1687-1847-2012-178

Cite this article as: Kurulay: Approximate analytic solutions of the modified Kawahara equation with homotopy analysis method. Advances in Difference Equations 2012 2012:178.

\section{Submit your manuscript to a SpringerOpen ${ }^{\circ}$ journal and benefit from:}

- Convenient online submission

- Rigorous peer review

- Immediate publication on acceptance

- Open access: articles freely available online

- High visibility within the field

- Retaining the copyright to your article 